\title{
Investigation of the influence of the symmetry of membership functions in the fuzzy controller on the quality of MPPT regulation in the photovoltaic system
}

\author{
Ruslan Gimazov ${ }^{1}$, and Stanislav Shidlovskiy, ${ }^{1, *}$ \\ ${ }^{1}$ National Research Tomsk Polytechnic University, 634050 Tomsk, Russia
}

\begin{abstract}
The paper presents the results of the investigation of the influence of asymmetric membership functions in a fuzzy controller on the quality of MPPT regulation in the photovoltaic system. Comparison of the quality of fuzzy MPPT regulation with symmetrical and asymmetric membership functions is carried out. The results of the research showed the advantage of using asymmetric membership functions in the MPPT algorithm with fuzzy logic.
\end{abstract}

\section{Maximum Power Point Tracking (MPPT)}

MPPT - maximum power point tracking - one of the most effective ways to increase the efficiency of photovoltaic systems. For the implementation of MPPT digital devices are used. The devices analyze the current vs. voltage curve $(\mathrm{CvV})$ by certain algorithms and determine the optimal current-voltage pair providing the maximum output power.

Figure 1 shows an example of the current-voltage characteristic of a photoelectric module with a maximum power point marked on it.

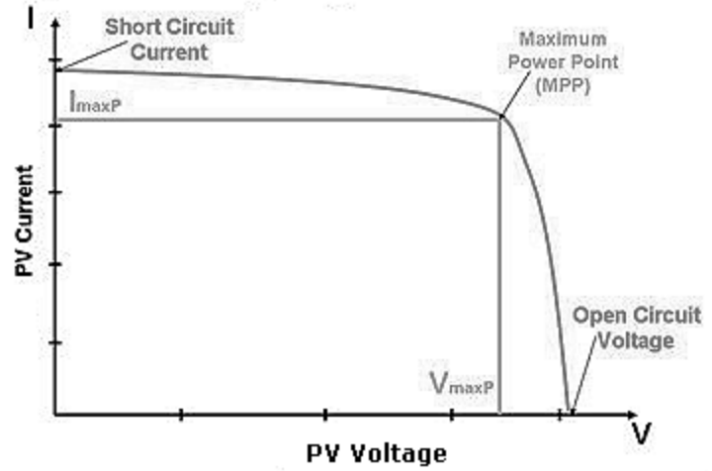

Fig. 1. MPPT on the current vs. voltage curve of the photovoltaic module.

\footnotetext{
*Corresponding author: shidlovskiy@tpu.ru
} 
The most common MPPT algorithm is the "perturbation-observation" algorithm. In this method, the MPPT device modifies the input resistance by a small value. As a result, the voltage of the solar installation varies. Calculating the electrical power and comparing its current value with the previous value, you can get the direction of searching for the point of maximum power.

The principle of the "perturbation-observation" algorithm on the power vs. voltage $(\mathrm{PvV})$ curve of a photocell is shown in Fig. 2.

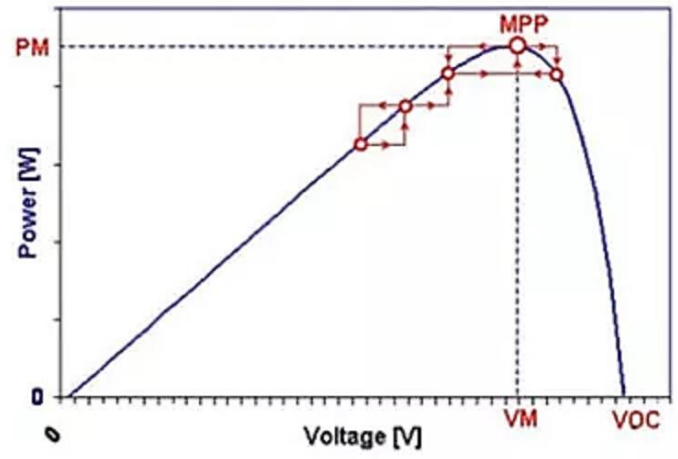

Fig. 2. The principle of the maximum power point tracking according to the perturbation and observation algorithm.

Disadvantages of the "perturbation-observation" algorithm are power fluctuations and a fixed time to performance. These problems can be solved with the help of adaptive algorithms, for example algorithms with fuzzy logic [1].

\section{Fuzzy logic}

The math theory of fuzzy logic allows you to generate fuzzy conclusions, through which more flexible control systems are implemented. It has been experimentally shown that the use of fuzzy logic in control (fuzzy control) gives better results, compared with those obtained with traditional control algorithms [2]. Figure 3 shows the principle of implementing a fuzzy controller.

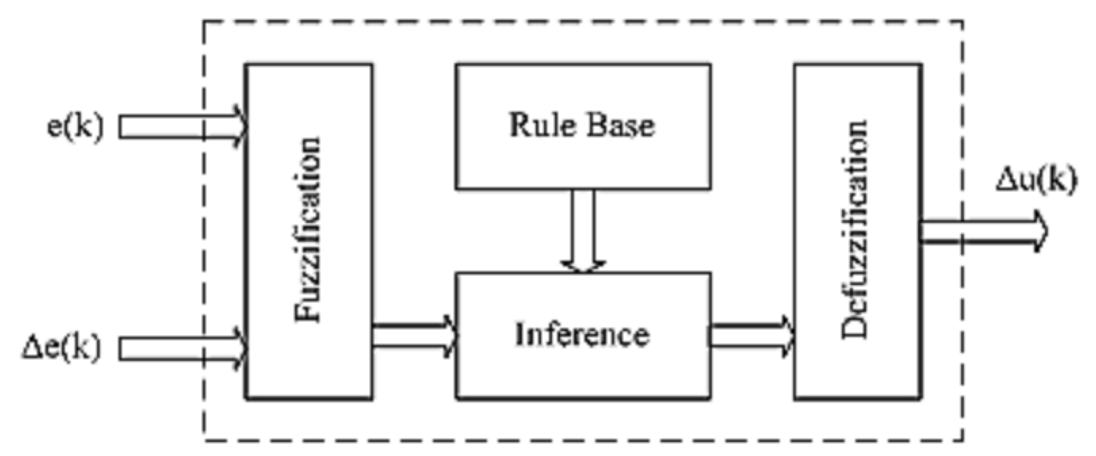

Fig. 3. Schematic diagram of the fuzzy controller.

A set of fuzzy input and output elements in the fuzzy controller database is regulated by the membership function area. In this paper, the influence of symmetric and asymmetric membership functions on the quality of MPPT regulation is investigated. 


\section{Investigation and conclusion}

To implement the study, a simulation model of photovoltaic system was developed [3].

The symmetry of membership functions in a fuzzy controller is determined by the shape of the fuzzy subset area. The shape of such area is determined by the base of fuzzy rules. The base of fuzzy rules for the system under investigation is presented in Table 1 .

Table 1. Rules of the fuzzy controller.

\begin{tabular}{|l|l|l|l|}
\hline $\mathbf{E} \downarrow \backslash \mathbf{d E} \rightarrow$ & low & middle & high \\
\hline low & low & low & low \\
\hline middleL & midL & midL & low \\
\hline middleH & midH & midL & low \\
\hline high & high & midH & midL \\
\hline
\end{tabular}

As input linguistic variables of the fuzzy controller, we use the ratio of solar power change to solar voltage change (E), as well as the behavior of $E(d E)$ :

$$
\begin{gathered}
E(k)=(P(k)-P(k-1)) /(V(k-V(k-1)) \\
d E(k)=E(k)-E(k-1)
\end{gathered}
$$

In the presented table, the values in the leftmost column and in the upper line define the functional affiliation of the input variables $(\mathrm{E}, \mathrm{dE})$. The remaining positions of the table determine the function of the output quantity, depending on the combination of the input values. If all the areas (subsets) of the membership functions of some of the variables are symmetric, then such a variable will be called symmetrically inscribed, and the membership function is symmetric. If there exists at least one area of the membership function that is not symmetric, then such a variable will be called asymmetrically inscribed, and the membership function is asymmetric. Figure 4 shows an example of symmetric membership functions. Figure 5 shows an example of asymmetric membership functions.

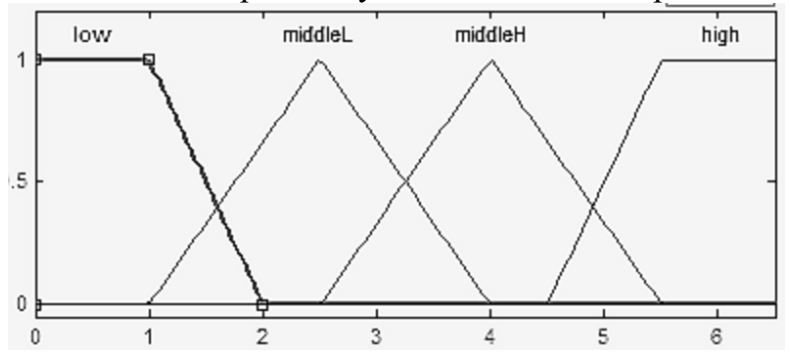

Fig. 4. Symmetric membership functions.

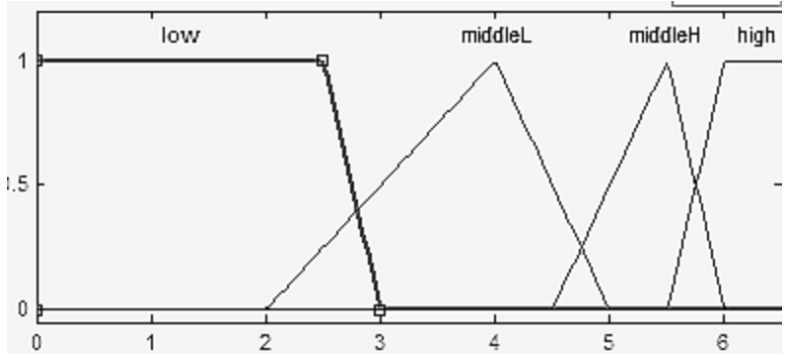

Fig. 5. Asymmetric membership functions. 
In the course of the study, comparative graphs of the process of searching for the maximum power point were obtained. Figure 6 shows the voltage variation graph using the "perturbation-observation" algorithm. Figure 7 shows comparative graphs of the change in the output power of solar cells during MPPT regulation.

The graphs indicate: 1 - MPPT algorithm without fuzzy logic, 2 - symmetrical fuzzy MPPT algorithm, 3 - asymmetric fuzzy MPPT algorithm.

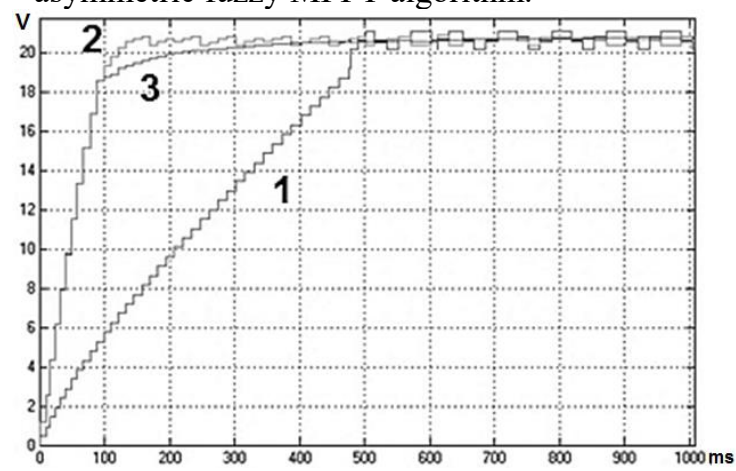

Fig. 6. Voltage variation.

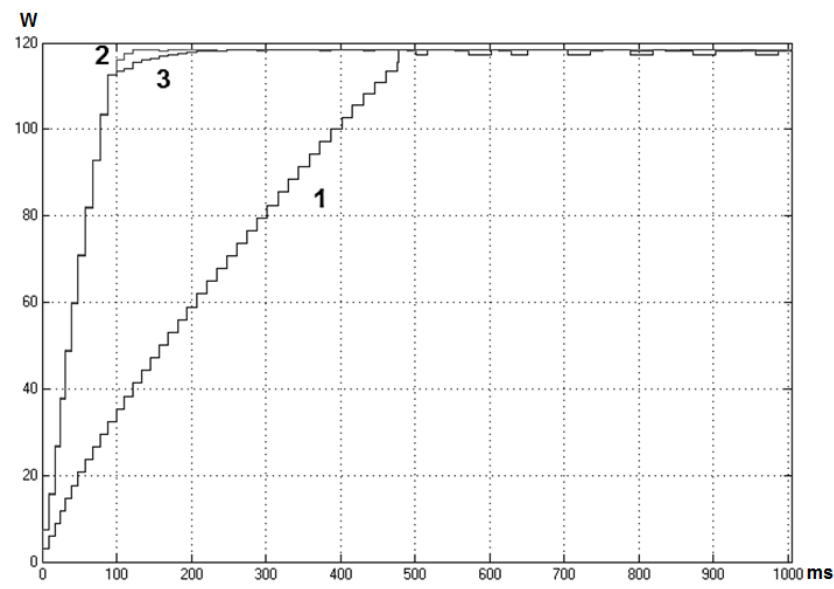

Fig. 7. Power variation.

A comparative analysis of the graphs shows that the use of fuzzy logic significantly improves the characteristics of the MPPT algorithm: decreases the search time for the extreme point, decreases the amplitude of voltage and power fluctuations. The use of asymmetrical membership functions to neutralize fluctuations in voltage and power. The use of symmetric functions minimizes the time of finding the extreme point.

\section{References}

1. S. Shidlovskiy, Automatic control. Reconfigurable structures (Tomsk State University, Tomsk, 2006)

2. K. M. Passino, S. Yurkovich, Fuzzy Control (Addison Wesley, Menlo Park, 1998)

3. S. Shidlovskiy, R. Gimazov, Cognitive Robotics, 1, 51 (2016) 